\title{
GOBIERNO UNIVERSITARIO
}

\author{
CONGRESO DE LA REPÚBLICA \\ Comisión de Educación y Cultura
}

\author{
Ponencia \\ DR. MANUEL PAREDES MANRIQUE \\ Rector de La Universidud Nacional Mayor de San Marcos:
}

\section{Conversatorio: Bases para una nueva Ley Universitaria}

El tema del Gobierno Universitario es evidentemente el punto central de cualquier ley universitaria; de hecho, la existencia de und ley se basa en la necesidad de normar el gobierno de las Universidades; en tal sentido, las exposiciones a las que asistimos nos dan una idea de la diversidad de perspectivas desde las que se puede tratar este tema.

La Universidad, no debemos ol vidar, es una institución de origen medieval, que se ha ido adaplando al carácter democrático de nuestra sociedad, y por tanto los enfoques exclusivamente empresariales, aunque estén matizados o encubiertos por las supuestas necesidades y ventajas de esta perspectiva, no deben ocultarnos und realidad; y es que si la Universidad se convirtiera en empresa, dejaría de ser Universidad. Los modernos paradigmas y los gurús de la moderna administración nos quieren obligar a pensar que el mercado es la panacea para todos los problemas, que la eficacia y eficiencia en el manejo empresarial se pueden trasladar íntegramente a cualquier actividad humana, y en este extremo no concordamos. La absoluta libertad de cada Universidad para determinar su forma de gobierno, haría innecesaria la existencia de la Ley, salvo para formalizar esa absoluta libertad.

Se olvida en ese extremo que la Universidad es una institución social; su existencia sólo tiene sentido dentro de una sociedad organizada, y las atribuciones y pri-

\section{Correspondencia}

Dr. Manuel Paredes Manrique

Reciorado UNMSM

Av. Repriblica de Chile 295

Lima 1 - Perí vilegios de que goza son concesiones de la sociedad organizada para que pueda cumplir mejor sus funciones. En tal sentido, la necesidad de determinar, aunque sea de manera general, su forma de gobierno, es um derecho de la Sociedad Organizada, vale decir del Estado. No se le puede atribuir el mismo carácter cue el de una empresa donde los dueños son los responnables de su luncionamiento, pues no se trabaja con bienes y servicios que el mercado regulará, sino que se trala de la vida y esperanza de los jovenes de nuestra sociedad y de mantener un nivel de calidad en la futura dirigencia del país. contribuyendo al desarmollo de éste. No puede existir institución con mayor responsabilidad social que aquella que forma el futuro de los ciudadanos. Ésta es la Universidad, y por consiguiente no puede asimilarse su forma de gobierno a la de un dueño o empresario privado.

Adicionalmente, cuando hablamos del grobierno de la Universidad debemos colocarmes en la perspectiva Latinoamericana, ya que este mismo tema desde la perspectiva Europea o Norteamericana cs totalmentc diferente; lo que hace la diferencia cs que nosotros tuvimos la revuelta juvenil de 1918, es decir un acontecimiento de hace 80 años y que fue el inicio del cogobierno Universitario. Ésta era una reaccion adccuada a la época y a las circunstancias, peróstas han variado nolablemente. Sin embargo, si encontramos un punto común entre las distintats exposiciones, es que escogieron ol tema del cogobierno como punto central de la propuesta, bien sea limitándolo, organizándolo o bien eliminándolo.

Hemos escuchado algunas propucstas concretas, pero detrás de cada una de ellas existe un concepro de Universidad, y si no somos conscientes de ello, corremosel riesgo de no dialogar sino de momologar alternadamente, y en diferentes idiomas, con lo cual llegaremos a la conclusión que la mejor forma de gobierno es la que a cada uno le parece. 
En definiliva, la presencia invisible del cogobierno que levanla pasiones encontradas si gue vigente a 80 años Je su nacimicnto y si hien cslo es malural, debe tenerse una perspecliva más amplia, pucs el gohierno de las Unjversidades no deja de ser un instrumento para conseguir los fines de la Universidad y por lanto lo primordial es ponernoside acucrdosobre cuales soln esos lines y luego intentar diseñar una forma de goloierno que nos de las mejores posibilidades de conseguirlos.

Permátanme expresar mi convicción. afincada en mis 4() años de maestro: La Universidad es una realidad compleja, pero se ha hecho más compleja que lo necesario por intentar darle un gobicrno supuestamente dcmocrático, participativo y colegiado. Anre ello, la tentacion de un sistema (le gobicrno cuasi empresiarial es grande y seguramente nos volvería más eficientes; pero Ia Lniversidad cs más gue una cmpresa, o cll lodo caso cs llna empresa del conocimiento y por consiguiente, con lodas sus imperfecciones. los sistemas de gobierno que ahora criticamos están leñidos de un nivel de utopía, de ideal democrálico. que no creo imposible de conciliar con la moderna administración y lodas sus ventajas. Si consideramos a la conjunción de mastros y alumbos, que csludian e investigan permanentemente comoun fin en sí mismo, que producen benelicios concretox a la socjedad en el aspeclo cientílico humanístico y tecnológico, nos estaremos accrcando al carácier y sentido de la Universidad, cl cual ha persistido por más de 800 años y gue hace de este mencster una de las más humanas actividadcs.

En lo que respecta a las propucstas de los distinguiJos expositores, lengo cercanías y cordiales lejanías, y eso cs nalural; no pucdo menos que concordar con la Dúsrpueda de la eficiencia en la gestión administrativa y la excelencia en los resultados educativos, dunque cso me obliga a pregumlar, si con cllo dehcmos dejar de lado la idea del accesor amplio de los estudianter a la Universidad y sin estar sesgado por factores económicos. Otrás propucstas son un lanto inmediatistaso por decirlo de otra maneral reformistas: no se debe anular el cogubicrno y sc debe tener un más ampliu malrgen de liberlad para las csirucluras de gobicrmo. Como se nutará, cn el fondo contimúa la inferrogante, no existe un consenso sobre la validez de la intervención del Eslado ch el inodo de gobicrno de las Unjversidades. Nadie duda del carácrer sociall de la Liniversidad, pero parece que será dilícil cncontrar el punto de ecjuilibrio del nivel en que debe intervenir el Estado.
Promulgar una ley que ralle de conciliar lodas las posicioncicon respecto al sistema de grobierno Universilario equivale a no bacer unal ley. Cren que lo gue nos convocá ch cste recinto es la lïme erecncia de la necesidad (le una ley. aumyuc, como cs uso democrático, mo sadistaga al lodos.

En 15 días me corresponderá compiritr con usledes en forma más amplia algunas de las idcals cue sobre el gobicrno de la Universidad me han suscilado, inicialmente en mis años en la docencia, lucgo ch mi permanencia como Decano y actualmente diriegiendo la Universidad de San Marcos.

\section{Conversatorio: Situación y Perspectivas de la Universidad en el Perú}

El gobicrno de las Universidades es sil duda uno de los temas nás imporłantes para la próxima Ley Universitaria. por cuanto cs un elcmendo lacilitador para lograr una Insitución Universitaria moderna, cliciense y capaz de alrontar con éxito los relos que la Educacion Superior enfrentarácon el inicio del mevo milenio.

El tema del gobierno, en lodas las leyes miversilarias alloriores, ha sido tratado en lorma polílica para complacer a todos los secteres de la vida Universitaria; de allísus caracteristicas gencrales: Representación por estamentos, representacion de mayoria y minoría de docentes y estudiantes, y grandes asambleas parlamentaristas. De esals mismas caracterislicas se desprendon

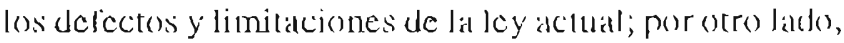
esta ley norma indistintamente paral Juiversidader de gestión privada o gestion estatal, generalcs o especializadas, grandes o pequcĩas.

Se ha señalado yá en anteriores oportunidades yue la eliciencia cs el Norle al cual sce clehe aspirar col la gestioin de las Linjersidader: la Nación espera resulaldos, la Sociedad necesita Uaiversidades generadoras de conocimiento, lamsmisorás del mismon y cluc criten vinculadis a la realidad de nuestro sistemal social y ecomómico; por ello es clue. si recomocomos la diversidald y pluralidad de nuestro pals, debemus lambićn normar cue se rcspete esid misma diversidad y pluralidad col las instituciones Universilarias.

El Decreto Supremo N" 882, que mormal la inversión privada en la educación, reconoce una realidad: La educación privada existe en el Perú. y sta razón de ser, leğí- 
tima por cierto, como en toda actividad privada, es el lucro; pero al mismo tiempo debemos señalar que no debe caerse en el nuevo error de igualar a todas las Universidades y considerarlas a todas como si fueran empresas privadas.

La Universidad Privada funciona bajo normas generales, con la libertad de gesción que ya le ha dado el D.S. 882, pero debe estar bajo la supervisión del Estado a través del Ministerio de Educación, pues éste no debe abdicar de su responsabilidad de velar porque los profesionales a quienes se otorgat título a nombre de la Nación y que luego servirán al país, sean realmente idóneos.

En el caso de la Universidad Pública, se le debe dolar de un sistema de gobierno ágil y eficiente, para que pueda competir en igualdad de condiciones con las Universidades Particulares que funcioban como empresas privadas, al menos las de reciente formación. Por tanto, el tema del gobierno de las Universidades al que voy a reterirme fundamentalmente, es el de la Universidad Estatal; es decir, aquella en la que el Estado invierte, aunque esa inversion no cubra el total de sus gastos, ya que cada vez los recursos que se asignan a la Universidad Estatal son menores en relación a sus necesidades, por lo que Esta se ve obligada a incrementar los recursos propios. Hago esta precisión porque una falacia constantemente repelida es que "el Estado dilapida su dinero en Universidades de baja calidad, masificadas y politizadas"; y esto no es cierto, aunque se pinte una imagen que muchos desean mantener comoun estigma sobre la Universidad Pública.

E) concepto de Universidad Estatal va mas allá de la condición que sea el Estaún el aportante de dinero; bien podría el Estado (y lo hace) destinar recursos a una institución privada, por necesidad social, y no por ello ésta. se convierte en una Institución del Estado. Lo que le da el carácter de pública o estatal a la Unjversidad, es que expresa la responsabilidad del Estado de brindar igualdad de oportunidades y acceso a la Educacion Superior a todos los peruanos independientemente de su condición económica.

Es también evidente que aquellas Universidades que estamos en proceso de Reorganización, y por tanto sujetus a un gobierno Universicario de excepción con comisiones de Reorganizacion ( $y$ no de intervencion como suelen decir algunos), tenemos und ventaja muy grande sobre las Universidades que no sc encuentran con este modelo de gestión. Quienes hemos vivido las Asambleas Universita- rias tradicionales: masificadas, politizadas y donde primaba el interés político o de grupo por encimar del jnterés de la instiación, notumos la gran diferencia. Lomismo se puede decir de los Consejos Universitarios. supuestamente organos ejecutivos, gerenciales deberíamos decir, pero en los cuales tanto por el número de sus integrantes así como por los diferentes intereses que representan, los acuerdos y avances eran muy jimitados.

No estamos abogando para que el sistema de las Comisiones de Reorganizacion se exlienda, una reorganización es por definicion temporal y luegon debe regresar a un sistema estable pero que conserve, preserve y perfeccione lo positivo de la Reorganivación y, por cuué no, corrija algunos errores si los hubiere. Este preámbulo es el fundamento para una propuesta de gobierno para las Universidades Estatales que a continuación desarrollaremos.

\section{NIVEL INTERUNIVERSITA RIO}

En el actual sistema. no existe un organismo que por encargo de la Sociedad ejerzal las funciones de evaluación y acreditación de Universidades. Lal Asamblea Nacional de Rectores que reline a todas las Lniversidades, Privadas y Estatales, generales y especializadas, grandes y pequeñas, no cumple ninguna de estas tunciones, es sólo la que proporciona los Carnés Universitarios y entrega los formatos de diphomas, los lampones y la tinta para los diplomas que otorgamks, previo pago de Ins misinos.

No tiene sentido alguno que la Asamblea Nacional de Rectores siga existicndo, ya que eso perpeluaría los defectos que tiene su actual gestión que. ante la lalla de reales funciones que cumplir, desarrolla diferentes eventos: Conferencias, Seminarios, elc. que hien pudieran ser cumplidos por las propias Universjdades agrupadas entre sí a voluncad, sin crear una burocracia innecevaria y consumir recursos cue podrían entregarie a las propias Universidades Estatales. Nos gustaría mucho por ejemplo en San Marcos contar con el hermoso audilorio que tiene la ANR, al igual seguramente yue oürds Universidades, pero esos recursos son destinados a una Asamblea que no cumple función vital alguna.

No planteamos que desaparezca cualquier vílaculo entre Universidades, y se conviertan cadda mal cn una isla sin supervisión, lo que planteamos es que el Estado astuma directamente su rol tiscalizador y normativi). Para 
cumplir con ello se debe recordar que la Universidad es la continuidad de la educacion básica, y por lanto le corresponde al Ministerio de Educación esta responsabilidad; por ello, planleamos la creacion del Consejo Nacional de Educación Superior, que podría tener la siguiente composición:

Ministro de Educación (s) Vice Ministro de Educación Superior (Presidente).

- Tres Rectores de las Universidades Estatales (los que rotarían cada dos añoss).

- Un Rector de las Universidades Particulares (rotaría cada dos años).

- Un representance del Consejo de Ciencia y Tecrología.

Estc Consejo tendría potestad para establecer las normas para la creación de nuevas Universidades, las que rijatn el funcionamiento de las nuevas Universidades, su cvaluacion, acreditacion; del mismo modo, que rengan iniciativa para la formulación de dispositivos legales relativos a la Educación Superior. Para estos cfectos conLaría con órganus que le apoyen y presten asesoramiento, de modr) que sus decisiones tengan un sólido basamento.

Igualmente asumiría funciones de la ANR, tales como: cmisión del Carnel Universitario. registro único de Graduados y Titulados, etc., así como dispondría del local e jnstalaciones de la ANR.

Las Universidades Estatales, tal como en alguna forma lo vienen haciendo, podrían establecer otras formas de cooperación entre cllas, es nuestra opinión que deberían formar una Asociación de Universidades Estarales o Consorcio ya que todas jas que tenemos una gestión Estatal posecmos problemas comunes, $y^{\prime}$ cl intercambio de experiencias y la cooperacion siempre será beneficiosa; pero no podemos pedirle a las propias Universidades que formen un órgano que se norme a sí mismo ya que esto es tunción del Estado.

\section{NIVEL DE ASA MBLEA UNIVERSITARIA}

La Asamblea Universitaria de la actual Ley se hasa en la representación de estamentos. Pero vicinsamente a los tres clásicos: docentes, estudiantes y egresados, se le añade, en la práclica, un cuarto estamento, el de las autoridades: Decanos, Directores de Post Grado y otros semejantes, los que pierden su condición de docentes y se convierten en "Autoridades", El número de Asambleíslas varía entre no menos de 6() y 120 representantes (ejemplo: Universidad Federico Villarreal 1()3, UNMSM 115); y como se dijo en la reunión anterior. las aukriodades son consideradas la patronal concepto absurdo ya cue la Universidad es del Esiado y por tante) de ledess, peros sin embargo, y en eso coincidirán conmigo los clue lian participado en Asambleas Universiarias, cn la práclica las Asambleas funcionan de esa manerd.

Por otra parle, la Ley indica que la Asamblea es el órgano máximo de gobierno de la Universidad. en una concepción parlamentarista clue no opera en ninguna de las Universidades más destacadas del mundi. las cuales tienen como su más importante brgano de gobierno a los Consejos Educativos de los guhiernos estatales (como en los Estados Unidos) o las Comisiones Educativas de los Landerso gobiernos regionales (como en el caso de Alemania).

Este gobierno parlamentarista no tiene sentido al guno y es el que ha conducido a excesos y rémoras en el crecimiento y' desarrollo de la Universidad. En la actualidad. la Ley le otorga funciones que no cumple y yue en la práclica se reducen al cubilelco para la clección del Rector y Vice Rectores. En particular, y a modo de ejemplo, en San Marcos nuestro Escallu(s) Ie da amplias prerrogativas a la Asamblea, ente ellals la de ralilicar diversos acios del Consejo Universilario. y rambien la de moditicar el propio Estatulo, y lo linico que sic ha obtenido en fos años de vigencia de la Ley y el Estatulo es la obstaculización. por razones políticas o de interés grupal, de medidas en muchos caisos lavorables, al mismo tiempo que no ha realizado ninguna de las muy necesarias moditicaciones estaturarials. que recién en nuestro períndo de reorganización hemos podido efecillar. Un solo cjemplo muestra los extremos de esta sifuación: el Reglamento General de lá Universidad debiera ser ratificado por la Asamblea Universirariar y évila a pesar de haber cambiado su conformación, de haberse renovado y reunido múltiples veces, jamás se puso de acuerdo y por tanto en más de doce años de vignencia del Extaluto, la Unjversidad no ha contado conl un Reglamento Generál.

Es evidente que la gestión de una Universidad no puede encargarse a un organo tan numeroso, tan diverso y que es fundamentalmente político en el más amplio sentido de la palabra, por ellu planteamos dos alternativas: 
Primera: que debe estudiarse con cuidado, es que la Asamblea deje de existir y que sus limitadas funciones sean totalmente asumidas por el Consejo Universitario.

Segunda: que la Asamblea sea totalmente reconvertida y deje de ser un brgano de gobierno para convertirse en un Foro de discusion en el cual se arriben a lineamientos de polílica y proyecciones de desarrollo. pero dejando el verdadero manejo de la Universidad al sigujente 6rgano, cual es, el Consejo Universitario.

Como consideranos la desaparición de la Asamblea solo como una alternativa, continuaremos en la exposición como si esta existiera, pero con las nuevas atribuciones que le hemos asignado; en dicho caso, es imperativa la desaparición de la representación por estamentos.

Los docentes conforman un solo estamento y la representación de principales, asociados y auxiliares en lislas separadas no tiene sentido. Por ello, planteamos que los miembros docentes de la Asamblea se presenten en listas únicas, independientemente de su categoría, y sean elegidos por todos los docentes en forma libre y directa.

En cuanto a los estudiantes, su participación puede significar un aporte, pero la proporción en que están incorporados en la actual composición de la Asamblea es excesiVals por lo que debe limicarse su número.

En tercer lugar, la Asumblea debe ser poco numerosá y sin limitaciones al número de docentes que pertenezcan a cada Facultad; así por ejemplo, si en una Universidad el $30 \%$ de los profesores pertenecen a la Facultad de Derecho, es evidente que esta realidad concreta debe plasmarse en su representación en la Asamblea y no tratal de medializarla con cupos que tergiversan la realidad. Si lo que se pretende es una verdadera democracia, que es el gobierno de las mayorías, debe darse cauce para que la mayoría tenga su adecuada representatividad.

Pero, ¿cómo determinaríamos el número de Asambleístas? Nuestra alternativa es que la Asamblea no) debe tener inćs de 50 representantes y los que correspondan a cada Facultad se determinarán por la proporcion que tenga el númcro de profesores de esia Facultad con relación al número total de profesores que tenga la Universidad. Así, en una Universidad con 1000 prolesores donde 300 sean de la Facultad $X$, por una regla de 3 simple le corresponderá tener 15 representantes y así sucesiyamente. En cada Faculad. determinado el número de representantes que le corresponde. sc presenlarán listas con ese número de candidatos conformados indistintamente en la proporcion que cada lista considere conveniente de profesores principalcs, asociados y an xiliares. La lisia ganadora tendrá el numero (o) al de representantes que le corresponda a esa Facultad.

Me adelanto a algunas probables objeciones:

"La Universidad será conducida por los profesores auxiliares ya que ellos comshimsen la mayorial.".

"Las Faculades pequerias en 'mimero de profesores" no tendrám represencatividad en la Asanblea"

A lo primero, debemos precisar que en al srunas Universidades esa "mayoría de prolesores auxiliares" no es" tan cierta, pues como se continúa con el vicio de oforgar la catcgoría en lunción del ticmpo de servicio y cumpliendo algunos requisitos, som cada vez más los principales. En algunas Universidades, la pirámide docente eslá inverlida; pero además debemos recordar que ésle es un órgano para señalar la política general de la Universidad, no pará conducir la gestión. ésa es función del Consejo Universilario. y por tamto no implica la necesidad de lítulos académicos o experiencia protesional en una disciplina determinada, sino volumiad de trabajo y experiencia, pero en liderazgo y conducción de organizaciones.

En cuanto a lo segundo, la posibilidad de cue no eslen representadas algunds Facultades "pequeñas", es cierta, pero es realista: si una Universidad rene lo Facultades, pero una de ellas, probablemente la mas meva, no tiene sino un $5 \%$ del rotal de protesores de la Universidad, no lendrá un miembro en la Asidmbled, pero, ¿para qué lo necesila? En la Asamblea se verán temas generales en su conjunto, no los asuntos que correspondan a la Facultad propiamente dicha, y por tanto debemos desterrar el mito de la omni representalividad: Que en lodos y cada um de los organos de la Universidad cstén representados todos los gruposi sectores y secciones de la Universidad. Pareciera clue en una concepción aristotélica se quisicra reflejar el macromundon en una multitud de micromundos. Esi) nu dehe ser así, cada cosa en su lugar.

¿Qué ventajas tiene esta Asamblea? Primero, es más ágil, sólo 50 representantes; segunuo, es más homogé- 
nea y licne funciones propias de una Asamblea, como es la de dar nurmas gencrales, detinir políticas de desarroIlo a largo plazo y olras semejantes, pero ya mo liene la lunción política de elegrir al Rector y Vice Reclores.

\section{NIVLI, DIE CONSEIO UNIVERSITARIO}

El Conscjo Universitario, de acuerdo a Ley, csilá conformado por los Decanos yuc represemall a su Fidultad, Ios Vice Recures, los alumnos: el gradundo y el Rector yue lo preside; en las Universidades Estatales el número Hultúa de 18 a 36 miembros. En la Liniversidad Federico Villarreal cslá confirmada por 33 y en San Marcos por 36 micmbros, de los cuales un tercio son esfudiantes. Sus finciones corresponden a las de un organo ejecutivo y de dirección de la Universidad; sin embareno, por el excesivo número de sus micmbros no llegal a cumplir cabalmentesu cometido. Los Decinos tienen por responsabilidad y lunción principal, su propia Facultad, están más imbuidos de la problemárica de ésta, que de la Universidad. y sin embargo, cstán obligados a entirentar cl manefo de loda la Universidad, y deban lomar decisiones en hase a la poca intormación de la que disponen en una sesión, o para cvilar esto, pedir tantos intormes que hacen improfuctivals las sesiones.

Si ponsanus ch un órganu ejeculivo, ésle debe estar comformado por un menor múmero de miembros, El Consejo Universilario licne cuc abocarsc fundanentalmenle a decisioncs y aclividaldes commes a loda la Universidad. como son: diclar el Reglamento General de Ia Universidad. Reglamento de Elecciones y otros Reglamenos internos; aprobar el presupuesio general de la Universidad; alloriwar hos aclos y contralos que alañen a la Universidad y resolver lodolo perinente a su economba, incluyendo la contabilidad y la liscalización; la creación. lusión, supresión o reorganización de sts unidades alademicas a administralivas; comlerir los grados aciadémicos y los lítulos prolesionales aprobadex por las Faculades, así como olorgar distincjones homorilicals y reconocer y revaljdar los cstudios grados Y lítulos de universidades extranjeras cuando la Universidad cilá allorizada paral hacerlo; aprobar anualmente el numero de vacantes para los procesos de admisión; declarar en recoso lemporalmente la Universi(lad o cualquiera de sus midades cuando lats circunslancias lo requicran; cjereer en instancia revisura las medidas disciplinarias sobre úncentes. estudiantes y personal administrativo; mermar sobre el Sistema Únicro de Biblintecas, Sistema Único de Malricula, el Siste- ma de Grados y Títulosi el Sistema Informático de la Universidad y los convenios interinstilucionales.

Todo lo demás, lundamentalmenle col lo acaućmico. debe ser parte de la allumonía Facultaliva y por lanto. no se necesita an Consejo Universitario con lodos los Decanos; proponemos un Conseju con la lercera parle del lotal de Decanos de la Universidad. pero. ¿cuales Decanos'?, los que ellos mismos delerminen a lravés de una elección o representación alutónomal cur lo que lentdríamos un Consejo pequeño, ageril, con gran responsadbilidad y suficiente poder de decisión.

Cada Universidad, atendiendo a su tamaño. com. plejidad y características, determinará el uúmero de Vice Rectores que deba tener. El Reclur y los Vicc Reclores de este Consejo deben ser elegidus en borma directa por los profesores, cm la misma volación en yue éstos elijan a sus representantes a la Asimbloa Universilaria y para las aluturidades de sls Falduader. Los cstudiantes estarán represenlados en el Conseju Liniversitario en la proporción de un tercio del número de Decanos que lo integren.

Pongamos un ejemplo. loda Universidad tendrá por lo menos un Recior y dos Vice Recuores. el academico y el administrativo, lucgo, y a parlir de esto sic inician las variaciones particulares de curbal Insilución: Inal Universidad com una activa y abumbance relaciom de intercambio y couperación externa lal ve\% considere yue debal lener un Vice Reclarialo de Relaciones Interinstitucionales, o como lo hemos pensiade en Sim Marcos. un Vicerrectur de Post Grado, un Vicerrector de Investigación y un Vicerrecur de Astmmos Esiadianliles. Cada Universidad, alendicndo a su condición. $p()-$ drá determinar el tamaño de su Comscjo Lniversitario. Exta es la clave para ylue cada Universidad leltgal las herramientas para forjarse un gobicrme acorde con su realidad. Si una Universidad decide lormar Consejos multitudinarios será por su propia decision y eslarai en

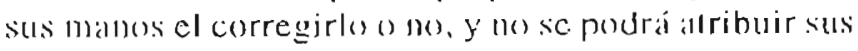
problemas de gestión a la Ley.

Esic punto me parece esencial pues es lo que mos daría a las Unjucrsidades Estatales. condicimes similares que a las privadas: las Universidades privadas pucden decidir sus órganos de gobierno y de gesilioll segrín sus necesidades y una Reorganizacion cs para cllas combs una sesión de directorio en la cual se cambien estos sislemas. En la Unjversidad Esialal el sistema rigidn normado por Ley dio lugar a yicios co algunas universi- 
dades y en otras felizmente no, pero como todo es un sistema regulado y estipulado por ley, no da berramientas para que la propia Universidad logre reorganizarse cuando lo necesite. Todos queremos Universidades Estatales eficientes y capaces de dar soluciones, no de provocar problemals que obliguen a medidas drásticas, pero absolutamente legales, como declarar en Reorganjzación a una Universidad. Si les damos las herramientas a las propias Universidades, esperemos que esas medidas no sean necesiarias en un futuro.

En particular, sobre el Consejo Universilario y el Vice Rectorado de Asuntos Estudiantiles, en San Marcos pensamos que debe existir en permanente coordinación con las orgamizaciones gremiales de los estudiantes, fundamentalmente para los aspectos que les compete como son: (arifas universitarias, equipamiento de bibliotecas; servicios de bienestar, etc.

\section{NIVEL DE CONSEJO DE FACULTAD}

Al igual que en el caso del Consejo Universitario y la Asamblea Universilaria, a nivel de cada Facultad se necesitan orģanos ágiles y con la suficiente autonomía $y$ alloridad; y tal vez con mayor razon, pues es und unidad más circunscrita. En la actual Ley se repiten a nivel de Faculad los mismos vicios que a nivel de toda la Universidad: un Consejo de Facultad Asumbleísta, con un sector de mayría y minoría de protesores principales; mayoría y minoría de asociados; representación de aluxiliares; representacion en mayoría y minoría de estudiantes, y la presidencia del Decano, quien a su vez es elegido por cl propio Consejo. Completan el cuadro en algunos casos, con voz pero sin voto, algunos otros funcionarios y docentes. Paradojicamente, los jetes de departamento y el Directoro Directores de Institutos de Investigación, que son los responsables de la vida académica cotidiana, no son integrantes del Consejo.

El sistemal, por las mismas razones que en el caso de la Asambled Universitaria, tiene el germen de la ineficiencia, la morosidad y la discusión bizantina. Repito, tiene el germen, ya que esto no ocurre necesariamente, pero en muchos casos, lamentablemente, así es: el Consejo de Facultad sc convierte en un campo de discusión en el cual los docentes, al no ser los responsables de los Departamentos, toman decísiones, o poco informadas o sin ninguna responsabilidad directa y posterior en la ejecución de las mismas.

El Consejo de Facultad con la conformación que hemos señalado se vuelve inoperante, sin capacidad de ejecular directamente sus acuerdos y silo (lispono de información mediatizada, y por tithto depende en exceso de la labor del Decano. Esor no la cunsideramos negativo, pueses el Decano el responsiable de la Facultad; lo que está mal, es que esic rol principal del Decano sea consecuencia de un Sistcmat ac Gobicrno complejo c incliciente. Pur otra parle, debcmos señalar que al igual que cn cl nivel de la Asamblea Universitaria, el Consejo de Facultad eligrc al Decano entre sus miembros o tuera de cllos, pero de lodas maneras este cargo crucial para cada Faculad. no es elegido en forma directa. Otra muestra más de la incoherencia entre el espíritu participativo y democráljos, y lo plassmado en la norma especilica.

Dada la conformación del Conscjo, sc corre el riesgo de "arreglos" entre una minorial de docentci con el tercio estudiantil, distorsionando muchas veces lal voluntad del clamstro expresada en las clecciones.

Consideramos que las Facultades deber ser dirigidas por el grupo de docentes cule estaín direclamente vinculados a la actividad académica cotidialna, por ello, plantealmosi una relormulación tulal de los integrantes del Consejo de Facultad; conformado por el Decano, Ios Directores de las Escuelas, el Director de Posi Grado (si lo hubiera), los jefes de Departamento, los Dircctores de los Institutos de Investigación, y otros directivos académicos, responsables en cada und de sus Unidades de la marclia de la Facultad. Ellos son los cue comocen directamente los problemas y pueden plantcar las soluciones. y si son aprobadas, las ejecutarán ellos mismos.

En San Marcos, ya contamosicon una experiencia piIoto que tienc la conformación que hemess mencionado para los Consejos de Facultad y: que hemos llanadu Consejos Acadómicos (CONAFA); poro hemos añadido un organo más cjecutivo al cyuc homos lhamado Comité de Gestión Académica (COGESA) y que está contormado por algunos de los micmbros del Consejo Académico: el Decano, el Secretario Académico, el Administrativo y el Director de la Unidad de Posi Grado de la Facultad. La experiencia es muy lavorable y mos ha permitido, en gran medida, desartollar una gestión más ãgil.

La participación de los estudiantes lambién debe ser reformulada. Los estudiantes sc agrupan cu un gromio por Facultad y tienen un sistema de organización por el cual eligen periódicamente a sus dirigentes, y por lanto, éstos son sus representantes; no requjiriendose clecciones especiales para representantes anle el Consejo de Fatcultad. A través de este Sistema, el Secretario de la Asociación de Estudiantes de dicha Facultad participa con voz y voto representando a su gremio. 


\section{SISTEMA DE ELECCIONES}

Como vemos, lodo el Sistema de gobierno se basa en elección directa, universal y secreta del Rector, Vice Rectores y Decianos, quiencs al ser autoridades elegidas goran de la suficiente autonomía y respaldo para gobernar la Universidiad en su conjunto y a nivel de las Faculades. En la eleccion de estas autoridades participarían docentes y estudiantes, correspondiendo a los primerus el $80 \%$ del peso electoral y a los segundos el $20 \%$, solo particinarían aquellos alumnos del tercio superior y a partir del segundo año de estudios.

A través del acto electoral se elegirán:

- Rector y Vice Rectores, según las listas generales que se presenten a njvel de toda la Universidad (participan docentes y estudiantes);

- Decanos (participan docentes y estudiantes);
- Jefes de Departamento, Direclores de Escuela, de Institutos y Centros de Investigación y el Director de Post Grado de cada Facultad (solo docentes);

- Represcntantes docentes a la Asámblea Universitaria.

Con esta elección se conformarían alutomáticamente la Asambleal Universitaria, el Consejo Unjversitarior, los Consejos de Facultad y se cligirían a las attoridades que regirán el destino de la Universidad por cinco años.

La participación de los estudiantes cslá asegurada, en el nivel que corresponde, sin extenderla al labores y decisiones que son competencia de los responnables respectivos.

Hasta aquí hemos presentado nuestro aporte de lo que creemos debe ser el sistema de grobierno que se norme en la futura Ley Universitaria; lo cuuc nos ha orientado es buscar organos eficientes y cjecutivos, pero que al mismo tiempo mantengan y, aún más, acrecienten el aspecto democrático de la Universidal. 